\title{
Nuclide Imaging of Vascular Graft-Platelet Interactions: Comparison of Indium Excess and Technetium Subtraction Techniques
}

\author{
Thomas W. Wakefield, M.D., Bengt Lindblad, M.D., Linda M. GRahaM, M.D., \\ Walter M. Whitehouse, Jr., M.D., Shawn D. Ripley, M.D., Neil A. Petry, M.S., \\ Susan A. Spaulding, B.S., William E. Burkel, Ph.D., and James C. Stanley, M.D. \\ Department of Surgery, Division of Peripheral Vascular Surgery; Department of Medicine, \\ Division of Nuclear Medicine; and Department of Anatomy and Cell Biology; \\ University of Michigan Medical School, Ann Arbor, Michigan 48109
}

Presented at the Annual Meeting of the Association for Academic Surgery, Cincinnati, Ohio, November 10-13, 1985

\begin{abstract}
Indium-111-labeled platelet adherence to ePTFE thoracoabdominal vascular prostheses in a canine model $(n=10)$ was quantitated by (1) an indium-111 excess technique, contrasting graft radioactivity to that in a reference region, and (2) a technetium- $99 \mathrm{~m}$ subtraction technique, with radioactivity of circulating platelets eliminated by discounting background blood activity. Variation in graft thrombogenicity was provided by seeding six prostheses with enzymatically derived autologous endothelial cells, and implanting four prostheses without seeding. Grafts were imaged at 1,4 , and 6 weeks postimplantation, with platelet labeling using indium-111-oxine and red blood cell labeling using technetium- $99 \mathrm{~m}$. At 7 weeks grafts were excised and gamma activity was measured in proximal, middle, and distal segments. Luminal generation of $\mathrm{TxB}_{2}$ and 6-keto-PGF ${ }_{1 \alpha}$ from midportions of grafts was assayed. Indium-111 excess ratios at 6 weeks correlated with actual gamma activity of excised grafts (proximal $r=0.80, P$ $<0.01$; middle $r=0.73, P<0.05$; distal $r=0.48$, ns) but such a correlation did not exist for the technetium- $99 \mathrm{~m}$ subtraction technique $(r=-0.05,-0.25$, and 0.16 , in the three segments, respectively, all ns). The ratio of graft to aortic $\mathrm{TxB}_{2}$ production revealed a positive correlation with graft gamma activity $(r=0.87, P<0.01)$, and the ratio of graft 6-keto-PGF ${ }_{1 a}$ to $\mathrm{TxB}_{2}$ production also correlated with gamma counts $(r=-0.64, P=0.05)$. In this experimental setting technetium-99m subtraction analysis was an imprecise method of detecting graft platelet accumulation, whereas indium-111 excess ratios proved to be a more accurate method of quantitating vascular prosthetic thrombogenicity. (ब 1986 Academic Press, Inc.
\end{abstract}

\section{INTRODUCTION}

Indium-111-oxine-labeled platelet imaging is a noninvasive technique used to document prosthetic graft-platelet interactions in vivo. In an earlier study on thoracoabdominal double velour knitted Dacron bypass grafts in a canine model, disappearance of the nuclide image was documented to accompany decreased graft thrombogenicity [13]. In contrast to fabricated Dacron grafts, expanded Teflon (ePTFE) prostheses are known to exhibit less surface platelet reactivity, and the optimal method of imaging accumulation of platelets in these conduits is undefined. The purpose of this investigation was to assess the efficacy of indium-111 ( ${ }^{111}$ In) excess and technetium$99 \mathrm{~m}\left({ }^{99 \mathrm{~m}} \mathrm{Tc}\right)$ subtraction techniques as applied to imaging of ePTFE thoracoabdominal grafts with varying surface thrombogenicity.

\section{METHODS}

Ten adult mongrel dogs having a mean weight of $25.7 \mathrm{~kg}$ underwent placement of 10 mm i.d. $\times 30$-cm ePTFE thoracoabdominal bypass grafts (W. L. Gore, Inc., Flagstaff, Ariz.). Prostheses were initially anastomosed end-to-side to the infrarenal abdominal aorta, and after systemic anticoagulation with intravenous heparin $(150 \mathrm{IU} / \mathrm{kg})$, the proximal graft was anastomosed end-to-end to the descending thoracic aorta. Protamine sulfate $(1.5 \mathrm{mg} / \mathrm{kg})$ was administered at the conclusion of the graft insertion. In order to preclude obscuration of the prosthesis during imaging by radioactivity 
in overlying organs, the spleen and left lobe of the liver were displaced from the field of study by suturing the stomach to the diaphragm and tacking the spleen to the right side of the dog's abdomen. Animal care complied with the Principles of Laboratory Animal Care and the Guide for the Care and Use of Laboratory Animals (NIH Publication No. 80-23, revised 1978).

Autologous endothelial cells for graft seeding were derived from $10-\mathrm{cm}$ segments of both jugular veins removed at the beginning of the procedure. These vein segments were everted over 5-mm-diameter stainless-steel rods and rinsed several times in Hanks' balanced salt solution without calcium and magnesium (CMF BSS). They were then incubated in trypsin-EDTA $(0.1 \%$ trypsin in CMF BSS, $0.125 \%$ EDTA, pH 8) for $10 \mathrm{~min}$ at $37^{\circ} \mathrm{C}$ and then in collagenase $(630 \mathrm{U} / \mathrm{ml})$ in Hanks' BSS with calcium and magnesium at $\mathrm{pH} 7.2$ for an additional $10 \mathrm{~min}$. Endothelial cells were washed from the vessel by spinning the rod in culture medium. The endothelium was then pelleted by centrifugation at $50 \mathrm{~g}$ for $2 \mathrm{~min}$ and resuspended in $0.5 \mathrm{ml}$ culture medium. These methods generally yielded a total of 0.5 to 1.5 $\times 10^{6}$ endothelial cells from both excised veins, as sheets of 5 to 30 cells. In a randomized, blinded fashion, six grafts were seeded with endothelium and four grafts remained unseeded. All grafts were filled with blood for a period of $5 \mathrm{~min}$ to provide a protein coating on the luminal surface to enhance endothelial cell attachment. Culture medium with or without endothelial cells was then incubated for $10 \mathrm{~min}$ within the graft. As a final preparation step grafts were rinsed with $10 \mathrm{ml}$ of heparinized blood.

Platelets were labeled with ${ }^{111}$ In 1 week preoperatively for baseline platelet survival analysis, and again at 1, 4, and 6 weeks after graft implantation for image analyses as well as additional platelet survival determinations. The technique of ${ }^{111}$ In platelet labeling was modified from that described by Thakur and associates [12]. Whole blood, $86 \mathrm{ml}$, was mixed with an acid citrate dextrose solution and this mixture centrifuged at $200 \mathrm{~g}$ for $15 \mathrm{~min}$ to re- move erythrocytes and leukocytes. The supernatant of platelet-rich plasma was decanted and centrifuged at $1000 \mathrm{~g}$ for $10 \mathrm{~min}$ and the platelet-poor plasma supernatant removed and saved. The remaining platelet button was gently washed free of plasma by layering with $2 \mathrm{ml}$ of modified Tyrodes buffer solution at $37^{\circ} \mathrm{C}$ followed by decanting. The platelet button was then resuspended in $4 \mathrm{ml}$ of modified Tyrodes buffer and this mixture was incubated for 15 min with $500 \mu \mathrm{Ci}$ of Indium-111-oxine (Amersham Corp., Arlington Heights, III.). To remove excess ${ }^{111}$ In not incorporated into platelets, $2 \mathrm{ml}$ of platelet-poor plasma was added to the "11 In-labeled platelet suspension followed by centrifugation at $1000 \mathrm{~g}$. The supernatant from this step was removed and its radioactivity determined using an ionization chamber. The platelet button was resuspended once again in platelet-poor plasma and its radioactivity was determined. Labeled platelets were then reinfused into the dogs. The labeling efficiency in this study averaged $70 \%$ (range 33 to $91 \%$ ). Blood samples were taken $30 \mathrm{~min}$ after platelet reinfusion for calculation of platelet recovery, which averaged $39 \%$. Platelet aggregation curves in response to ADP were measured in samples before and after each labeling, as well as in recovery samples. Thromboxane $\mathrm{B}_{2}$ levels were measured using a modified radioimmunoassay technique in samples bcfore and after each labeling. Three methods, linear, gamma, and weighted mean analyses, were used for platelet survival calculations [1]. Blood sampling occurred daily over a 6-day period following labeled platelet reinfusion for platelet survival determinations.

Imaging was performed $48 \mathrm{hr}$ after platelet labeling with a gamma scintillation camera and consisted of an ${ }^{11}$ In platelet image, as well as a dynamic ${ }^{99 \mathrm{~m}} \mathrm{Tc}$-labeled red blood cell flow image (Fig. 1) and static ${ }^{99 \mathrm{~m}} \mathrm{Tc}$ image for blood pool determinations. Data from these studies were acquired and stored on a digital computer for analysis. Graft images were subdivided into three regions of interest (ROI) on the basis of the ${ }^{99 \mathrm{~m}} \mathrm{Tc}$ flow image and static flow scan: the proximal thoracic region, a middle region corresponding to the $5 \mathrm{~cm}$ immediately above the 


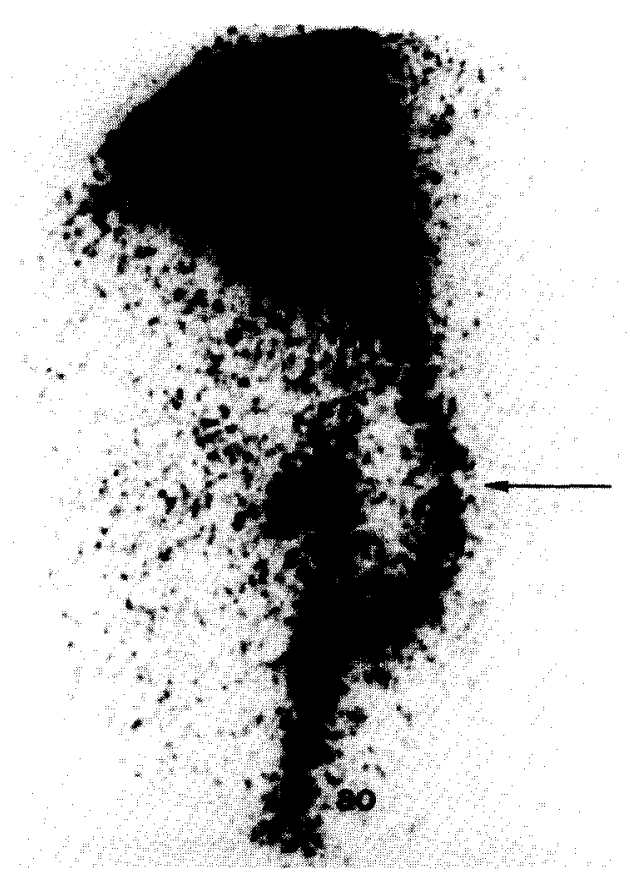

FIG. 1. ${ }^{99 \mathrm{~m}} \mathrm{Tc}$-red blood cell labeled-flow image of a thoracoabdominal graft (arrow). Native abdominal aorta (ao), mediastinal structures (m).

diaphragm, and a distal abdominal region. Two methods of image assessment were evaluated. The ${ }^{111}$ In excess ratio method compared the ratio of ${ }^{111}$ In counts in each of the graft's ROIs to the ${ }^{111}$ In counts over an abdominal aortic reference region (ARR) and was defined by

${ }^{111}$ In excess ratio

$$
=\frac{{ }^{111} \operatorname{In}(\mathrm{ROI})-{ }^{111} \operatorname{In}(\mathrm{ARR})}{{ }^{111} \operatorname{In}(\mathrm{ARR})} .
$$

The ${ }^{99 \mathrm{~m}} \mathrm{Tc}$ blood pool subtraction analysis [8] was performed on these same regions by defining the ratio of ${ }^{111} \mathrm{In}$ to ${ }^{99 \mathrm{~m}} \mathrm{Tc}$ in an aortic reference region and then multiplying the ${ }^{99 m} \mathrm{Tc}$ counts in each graft ROI by this ratio. This provided an estimation of ${ }^{111}$ In blood pool (BP) activity. The ${ }^{99 \mathrm{~m}} \mathrm{Tc}$ blood pool subtraction was then determined by subtracting the ${ }^{111}$ In blood pool from ${ }^{111}$ In activity in each ROI of the graft, and dividing the remainder by the ${ }^{111}$ In blood pool according to the formula
Technetium blood pool subtraction ratio

$$
=\frac{{ }^{111} \operatorname{In}(\mathrm{ROI})-{ }^{111} \operatorname{In}(\mathrm{BP})}{{ }^{111} \operatorname{In}(\mathrm{BP})} .
$$

The expcrimcnt was terminated 7 weeks following graft implantation, 7 days after the final imaging. At that time all dogs were heparinized $(150 \mathrm{IU} / \mathrm{kg})$ and after isolation and cannulation, the grafts were perfused with Dulbecco's phosphate-buffered saline before their careful removal. A central cylinder of graft, immediately above the diaphragm, was assayed for luminal production of prostacyclin and thromboxane during a 5-min incubation with Hanks' buffer, both with and without arachadonic acid stimulation $(20 \mu M)$. These prostanoids were quantitated by measuring their stable metabolic breakdown products, 6 keto- $\mathrm{PGF}_{1 \alpha}$ and thromboxane $\mathrm{B}_{2}$, respectively. The grafts were then opened longitudinally and photographed. Grafts were cut into $2-\mathrm{cm}$ sections and placed in glutaraldehyde. Radioactivity of these sections was measured in a gamma scintillation spectrometer. The results were corrected for the time and amount of ${ }^{111}$ In injected, as well as the exact surface areas of the sections measured. This correction took into account the 2.8 day half-life of ${ }^{111} \mathrm{In}$. Results were expressed as percent injected ${ }^{111}$ In $\mathrm{dose} / \mathrm{cm}^{2}$ of graft. Correlations among image analysis, direct gamma counting, and prostanoid production were then made. Statistical analyses of data included Student's $t$ tests and determination of correlation coefficients where appropriate.

\section{RESULTS}

Actual graft gamma radioactivity, reflecting accumulation of labeled platelets, correlated with ${ }^{111}$ In excess ratio measurements in a positive manner within the proximal and middlc segments of the graft $(P<0.01$ proximal, $P$ $<0.05$ middle; with correlation coefficients of $r=0.80$ and 0.73 , respectively). The correlation was not significant in the abdominal segment, $(r=0.48)$. These correlations compared 6-week images to 7-week graft counts. No correlation existed between actual graft gamma 
radioactivity and data derived from ${ }^{99} \mathrm{~m} \mathrm{Tc}$ subtraction ratio method $(r=-0.05,-0.25$ and 0.16 in the three above graft segments, respectively).

Assessment of ${ }^{111}$ In excess and ${ }^{99 \mathrm{~m}} \mathrm{Tc}$ subtraction data at 1 week, revealed: mean ${ }^{111}$ In ratio method, graft/abdominal aortic reference region radioactivity values of 1.12 (proximal), 0.46 (middle), and 0.33 (distal), while the values of graft excess radioactivity determined by the ${ }^{99 \mathrm{~m}} \mathrm{Tc}$ subtraction method were 0.31 (proximal), 0.39 (middle), and 0.18 (distal). At 4 weeks, similar values for the ${ }^{111}$ In ratio method in these graft segments were 0.96, 0.42 , and 0.35 , whereas the ${ }^{99 \mathrm{~m}} \mathrm{Tc}$ subtraction values were $0.35,0.68$, and 0.34 , respectively. At 6 weeks, the values for the ${ }^{11}$ In ratio method were $0.61,0.24$, and 0.16 , while the values for the ${ }^{99 \mathrm{~m}} \mathrm{Tc}$ subtraction were 0.24 , 0.26 , and 0.10 in these areas, respectively.

Comparisons of seeded to unseeded grafts revealed certain trends, although significant differences were not found. For example, seeded grafts exhibited a mean gamma radioactivity of $0.00165 \%$ injected ${ }^{111} \mathrm{In}$ dose $/ \mathrm{cm}^{2}$ of graft, while unseeded grafts had a mean gamma activity of $0.0025 \%$ injected ${ }^{111}$ In dose/ $\mathrm{cm}^{2}$ of graft. ${ }^{111}$ In excess ratios for seeded grafts

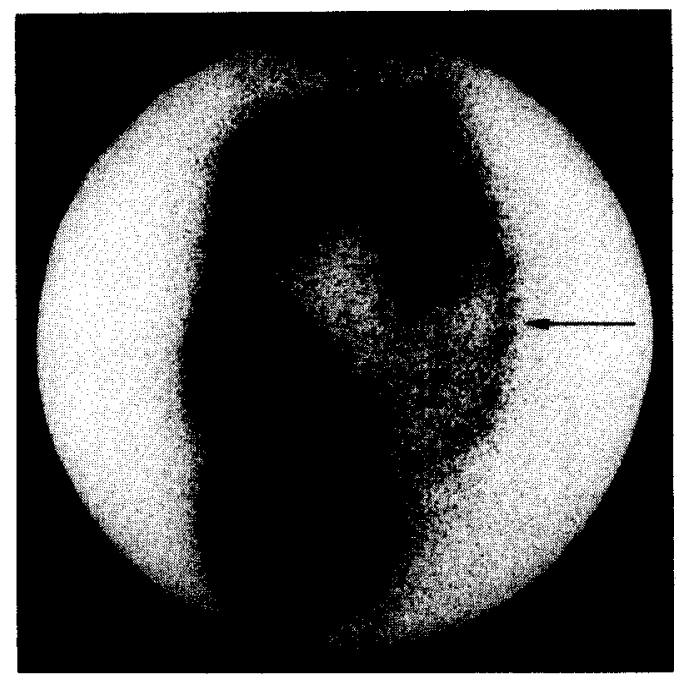

FIG. 2. One week ${ }^{111}$ In image of an endothelial cell seeded thoracoabdominal graft (arrow), liver (l), and spleen (s). at 1,4 , and 6 weeks were $0.44,0.47$, and 0.26 , respectively, while ratios for unseeded grafts at these same times were $0.94,0.74$, and 0.45 , respectively. Seeded grafts demonstrated a progressive loss of ${ }^{111}$ In activity at 4 and 6 weeks reflecting successful graft endothelialization (Figs. 2-4). This was in contrast to persistent ${ }^{111}$ In activity observed at 4 and 6 weeks in unseeded grafts, reflecting continued platelet deposition (Figs. 5-7).

Platelet survivals, using weighted mean analysis, revealed $81 \%$ normalization toward baseline at 6 weeks in the seeded group, compared to a $54 \%$ normalization in the unseeded group. Although a trend existed, these differences were not statistically significant. Gamma platelet survival analysis revealed 85 and $56 \%$ normalization in seeded and unseeded grafts, respectively. Linear calculations of platelet survival revealed even less of a separation between the seeded and unseeded grafts, with an 85 and $79 \%$ normalization occurring in these two groups, respectively. No correlation was found between platelet survivals and gamma counting with any of these methods.

The process of platelet labeling with ${ }^{11}$ In did not cause any significant elevation in thromboxane production, with the mean in-

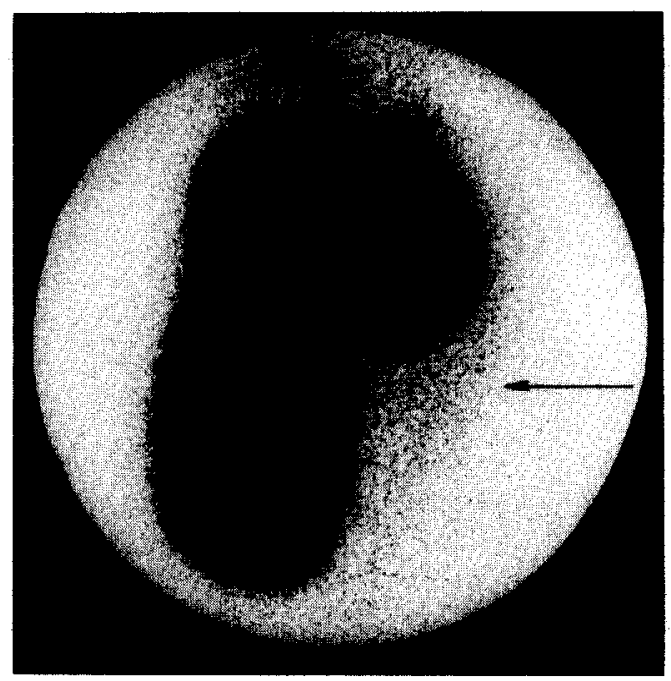

FIG. 3. Absent 4-week "1'In image of an endothelial cell seeded thoracoabdominal graft (arrow), liver (1), and spleen (s). 


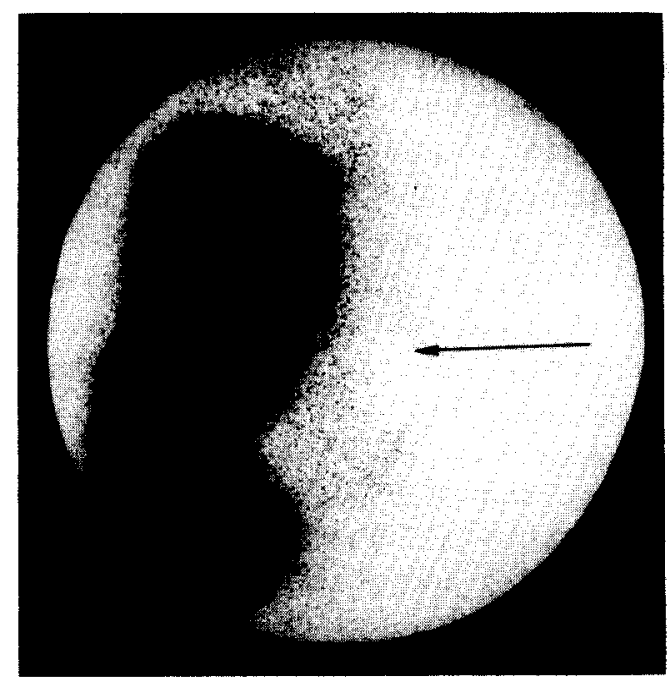

FiG. 4. Absent 6-week "'In image of an endothelial cell seeded thoracoabdominal graft (arrow), liver (l) and spleen (s).

crease in thromboxane $B_{2}$ measured in unlabeled to labeled platelet-rich plasma being $9 \%$, although a wide range was observed from -37 to $+85 \%$. The mean ADP-induced platelet aggregation of $35 \%$ in unlabeled samples was similar to that of 29 and $39 \%$ in the labeled and recovery samples, respectively.

The ratio of graft to aortic thromboxane

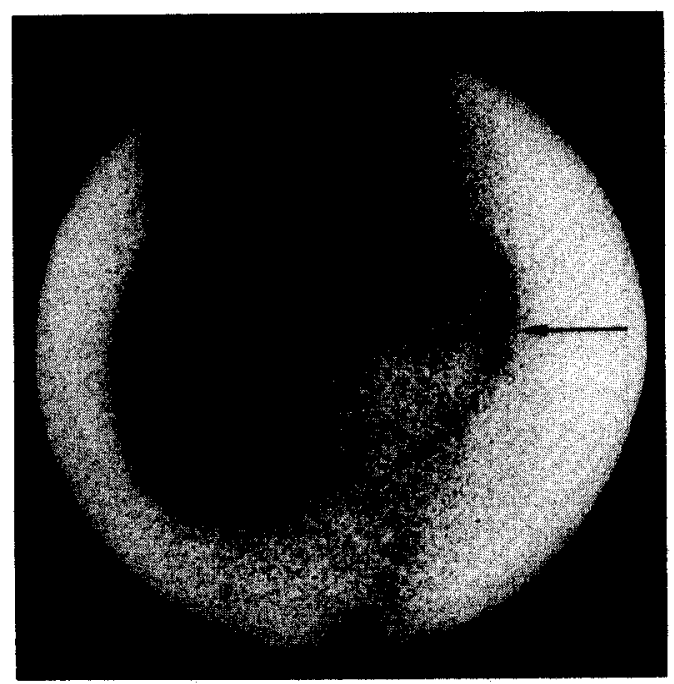

FIG. 6. Four week "11'In image of an unseeded thoracoabdominal graft (arrow), liver (l), and spleen (s).

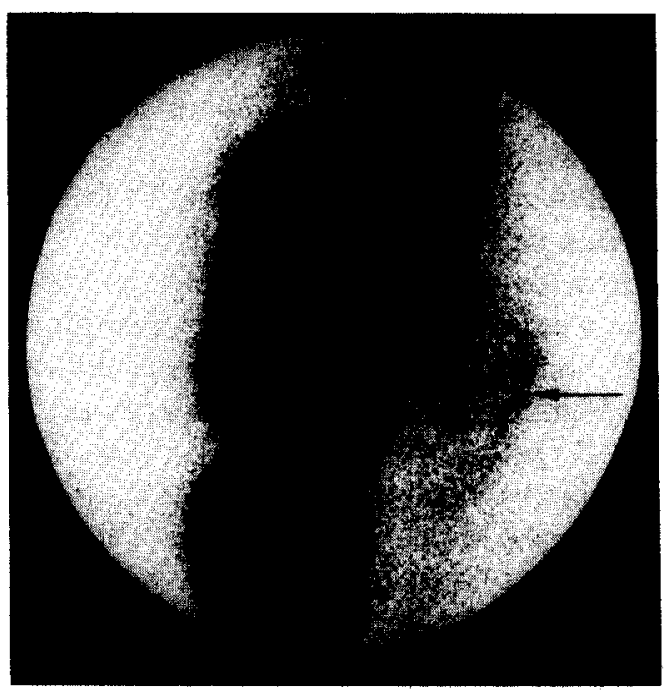

FIG. 5. One week ${ }^{111}$ In image of an unseeded thoracoabdominal graft (arrow), liver (l), and spleen (s).

generation correlated highly with absolute gamma counts in the graft midsegment (without arachadonic acid stimulation $r=0.87, P$ $<0.01$, and with arachadonic acid stimulation $r=0.85, P<0.01$ ). In each case the ratio of graft to aortic thromboxane was 1.5 to 1.0 . Absolutc thromboxane concentrations also correlated with absolute graft gamma activity

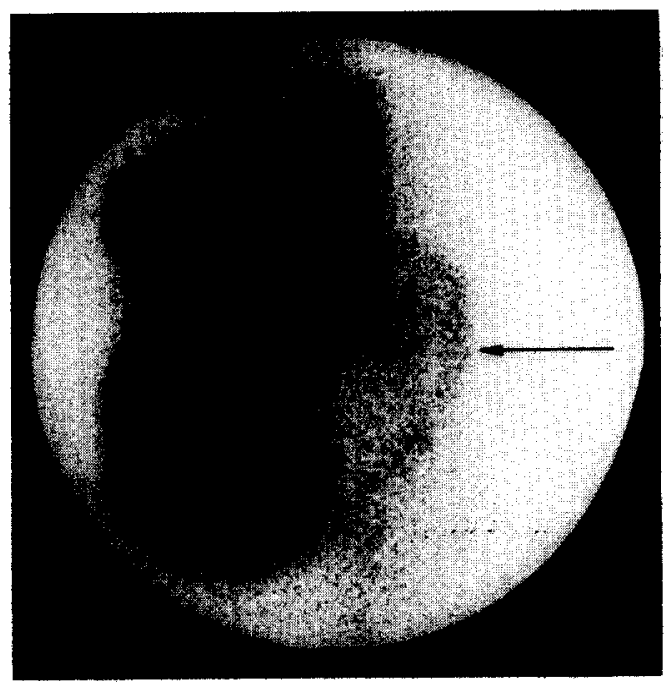

FiG. 7. Six week ${ }^{111}$ In image of an unseeded thoracoabdominal graft (arrow), liver (l), and spleen (s). 
(without arachadonic acid stimulation $r$ $=0.76, P<0.05$, with arachadonic acid stimulation $r=0.72, P<0.05$ ). In a similar manner, the ratio of prostacyclin to thromboxane generation by the graft midsegment without arachadonic acid stimulation correlated negatively with actual graft gamma radioactivity (the ratio being 0.27 to $1.0, r=-0.64$, $P=0.05$ ).

\section{DISCUSSION}

Diminished ${ }^{111}$ In platelet deposition on Dacron double velour prostheses has been demonstrated previously by the authors to reflect endothelialization of the graft [13]. The morphologic characteristics of endothelial seeded and unseeded EPTFE thoracoabdominal bypass grafts have also been documented by the authors [5]. In the latter studies, endothelial coverage in unseeded grafts never exceeded $10 \%$ of the luminal surface at either 2 or 4 weeks postimplantation, whereas seeded grafts demonstrated endothelial coverage of 64 and $91 \%$ at these time periods, respectively. A noninvasive method to evaluate this endothelialization, such as currently described, has clear value as an investigative procedure in the laboratory and in clinical practice.

The timing of graft imaging in our experiment deserves mention. Others have proposed that ePTFE grafts inserted into the femoral or carotid region when exposed to blood with unlabeled platelets for more than $1 \mathrm{hr}$ would not image for up to 3 days after labeled platelet injection, because subsequent platelet deposition and turnover was low [7]. Visualization of grafts has been noted to be maximal 48 to $72 \mathrm{hr}$ after injection. Differences in platelet adherence to ePTFE and Dacron have not been universally reported [9], although some have observed that ePTFE grafts take up platelets very slowly, at a rate comparable to native arterial autografts, and appear inherently less thrombogenic than Dacron [2]. We were able to image platelets on ePTFE surfaces successfully at 24,48 , and $72 \mathrm{hr}$ after reinjection of labeled platelets. In the current study imaging was recorded $48 \mathrm{hr}$ after reinjection, a time that seemed to offer the least amount of blood pool background while still being sensitive and specific for platelet deposition. Our ability to visualize grafts at this early time may be a reflection of the greater surface areas of the thoracoabdominal grafts used in this experiment.

${ }^{99 \mathrm{~m}} \mathrm{Tc}$ blood pool subtraction, which others have labeled "percent indium excess." [8], is useful when a suitable reference region such as a blood vessel of similar caliber and depth to the graft is not present in the field of the scintillation camera. This technique involves a ${ }^{99 \mathrm{~m}} \mathrm{Tc}$ blood flow image to compensate for the circulating blood pool ${ }^{111}$ In activity present at the time the gamma image is obtained. In our particular experiment, the ${ }^{99 \mathrm{~m}} \mathrm{Tc}$ reference was invalid because of overlying ${ }^{99 \mathrm{~m}} \mathrm{Tc}$ activity in the kidney and ureters in at least half the studies. Similarly, areas of the proximal and distal graft were frequently obscured by mediastinal and renal ${ }^{99 \mathrm{~m}} \mathrm{Tc}$ radioactivity, making accurate assessment with ${ }^{99 \mathrm{~m}} \mathrm{Tc}$ subtraction impossible. These difficulties may not arise in the evaluation of extremity vessels where overlying organs are not present to cause interference.

${ }^{111}$ In excess ratio analysis is derived very much like a thrombogenicity index [6]. The close correlation between ${ }^{111}$ In excess ratio analysis and actual graft gamma activity suggests that a direct measurement between ${ }^{11}$ In activity on the graft and a suitable reference region similar in size and distance from the gamma camera is most useful. However, in many clinical situations, a suitable reference region may be difficult to define. In these latter circumstances, with the limitations as expressed above, the ${ }^{99 \mathrm{~m}} \mathrm{Tc}$ blood pool subtraction method may be more applicable. Thus, the proper method of analysis depends on the ability to locate a suitable reference region.

Platelet survival studies have been performed for different grafts with varying degrees of endothelial coverage $[3,4,10]$. In unseeded woven thoracoabdominal Dacron grafts placed in dogs, acute platelet survival diminished significantly, returned toward initial survival values by 1 month, but even at 18 months remained less than normal. In 
knitted Dacron grafts seeded with endothelial cells, platelet survival returned to preimplantation values at 8 weeks. The current study with ePTFE grafts documented that platelet survival tended to return toward preimplantation values at 6 weeks, but never fully normalized.

Thromboxane and prostacyclin production has also been assessed in canine experiments with graft endothelialization. The balance between these prostanoids has been considered important in determining platelet deposition as well as long-term graft patency [11]. We observed that thromboxane production easily predicted absolute graft gamma radioactivity, indicating that graft surface platelets were still metabolically active. We also observed a negative correlation between the ratio of prostacyclin to thromboxane production and absolute gamma activity, indicating that graft regions covered with platelets did not produce as much prostacyclin as other areas.

${ }^{111}$ In platelet imaging provides a noninvasive technique for accurate assessment of platelet-surface interactions in ePTFE grafts. This technology will allow sequential study of vascular prostheses. ${ }^{111}$ In excess ratio analyses appear superior to ${ }^{99 \mathrm{~m}} \mathrm{Tc}$ subtraction analyses when a proper reference can be defined on the gamma image.

\section{REFERENCES}

1. Belcher, E. H., Berlin, N. I., Eernisse, J. G., Garby, L., Glass, H. I., Heimpel, H., Lee, M., Lewis, S. M., McIntyre, P. A., Mollison, P. L., Murphy, E. A., Najean, Y., Pettit, J. E., and Szur, L. Recommended methods for radioisotope platelet survival studies. By the panel on diagnostic application of radioisotopes in hematology, International Committee for Standardization in Hematology. Blood 50: 1137, 1977.

2. Callow, A. D., Connolly, R., O'Donnell, T. F., Gembarowicz, R., Keough, E., Ramberg-Laskaris, K., and Valeri, C. R. Platelet-arterial synthetic graft interaction and its modification. Arch. Surg. 117: 1447, 1982.
3. Clagett, G. P., Russo, M., and Hufnagel, H. Platelet changes following placement of aortic prostheses in dogs. 1. Biochemical and functional alterations. J. Lab. Clin. Med. 97: 345, 1981.

4. Clagett, G. P., Burkel, W. E., Sharefkin, J. B., Ford, J. W., Hufnagel, H., Vinter, D. W., Kahn, R. H., Graham, L. M., Stanley, J. C., and Ramwell, P. W. Platelet reactivity in vivo in dogs with arterial prostheses seeded with endothelial cells. Circulation 69: 632, 1984.

5. Graham, L. M., Burkel, W. E., Ford, J. W., Vinter, D. W., Kahn, R. H., and Stanley, J. C. Expanded polytetrafluoroethylene vascular prostheses seeded with enzymatically derived and cultured canine endothelial cells. Surgery 91: 550, 1982.

6. Goldman, M. D., Simpson, D., Hawker, R. J., Norcott, H. C., and McCollum, C. N. Aspirin and dypyridamole reduce platelet deposition on prosthetic femoropoplitcal grafts in man. Ann. Surg. 198: 713, 1983.

7. Megerman, J., Christenson, J. T., Hanel, K. C., Strauss, H. W., and Abbott, W. M. Imaging vascular grafts in vivo with Indium-1 11-labeled platelets. Ann. Surg. 198: 178, 1983.

8. Powers, W. J., Mathias, C. J., Welch, M. J., Sherman, L. A., Siegel, B. A., and Clarkson, T. B. Scintigraphic detection of platelet deposition in atherosclerotic macaques: A new technique for investigation of antithrombotic drugs. Thromb. Res. 25: 137, 1982.

9. Pumphrey, C. W., Chesebro, J. H., Fuster, V., Wahner, H. W., Dewanjee, M. K., Kaye, M. P., Hollier, L. H., and Pairolero, P. C. Quantitation of platelet deposition on human peripheral arterial bypass grafts. Circulation 64 (Suppl. 4): 66, 1981.

10. Sharefkin, J. B., Latker, C., Smith, M., Cruess, D., Clagett, G. P., and Rich, N. M. Early normalization of platelet survival by endothelial seeding of Dacron arterial prostheses in dogs. Surgery 92: 385, 1982.

11. Sicard, G. A., Allen, B. T., Long, J. A., Welch, M. J., Griffin, A., Clark, R. E., and Anderson, C. B. Prostaglandin production and platelet reactivity of smalldiameter grafts. J. Vasc. Surg. 1: 774, 1984.

12. Thakur, M. L., Walsh, L., Malech, H. L., and Gottschalk, A. Indium-111-labeled human platelets: Improved method, efficiency and evaluation. J. Nucl. Med. 22: 381, 1981.

13. Whitehouse, W. M., Jr., Wakefield, T. W., Vinter, D. W., Ford, J. W., Swanson, D. P., Thrall J. H., Froelich, J. W., Brown, L. E., Burkel, W. E., Graham, L. M., and Stanley, J. C. Indium-111-oxine labeled platelet imaging of endothelial seeded Dacron thoracoabdominal vascular prostheses in a canine model. Trans. Amer. Soc. Artif. Intern. Organs 29: 183, 1983. 